\title{
Lessons Learned or Forgotten? Impacts of COVID-19 on the Future Direction of Global (e-)Mental Health Care
}

\author{
D. Mucic ${ }^{1}$ J. H. Shore ${ }^{2,3} \cdot$ D. M. Hilty ${ }^{4}$ K. Krysta ${ }^{5} \cdot$ M. Krzystanek $^{5}$
}

Accepted: 1 September 2021 / Published online: 29 November 2021

(c) The Author(s), under exclusive licence to Springer Science+Business Media, LLC, part of Springer Nature 2021

\begin{abstract}
Purpose of Review The COVID-19 pandemic has impacted lives globally, posing unique challenges to mental health services exposing vulnerability and limitations within these systems. During the course of the pandemic, telecommunications technologies (e-mental health care) have served a critical role in psychiatric care. It is important to understand current lessons learned in e-mental health care and implications for global mental health systems for both emerging from the pandemic and after the pandemic has ended. Recent Findings There are significant regulatory, policy, and evaluation challenges for global e-mental health impacting patients, clinicians, health systems, and decision-makers. These include complex regulatory issues, difficulties of providing care across boundaries, and keeping pace with the implementation of new technologies in behavioral health.

Summary The collaborative development of global standards along with policies, appropriate regulations, and developing new models of research and development opens the possibility of improved access to care across national boundaries.
\end{abstract}

Keywords Digital psychiatry $\cdot$ Emergency response $\cdot$ COVID-19 $\cdot$ e-Mental health $\cdot$ Telepsychiatry $\cdot$ International health

\section{Introduction}

\section{Impact of COVID-19 on Mental Health (Care)}

The COVID-19 pandemic has impacted lives globally, posing unique challenges to mental health services around the

This article is part of the Topical Collection on Psychiatry in the Digital Age

$\triangle$ D. Mucic

dmucic@gmail.com

1 Little Prince Treatment Centre, Havneholmen 82, 5th, 1561 Copenhagen V, Denmark

2 Office of Telehealth and Technology Implementation for Behavioral Health Practice and Science (TIPS), Department of Psychiatry, Aurora, USA

3 Department of Psychiatry and Family Medicine, School of Medicine And Centers for American Indian and Alaska Native Health, School of Public Health, University of Colorado Anschutz Medical Campus, Aurora, CO, USA

4 VA Northern California Health Care, System \& UC Davis School of Medicine, 2230 Stockton Boulevard, Sacramento, CA 95817, USA

5 Department of Psychiatry and Psychotherapy, Faculty of Medical Sciences in Katowice, Clinic of Psychiatric Rehabilitation, Medical University of Silesia, Ziołowa 45/47, 40-635 Katowice, Poland globe exposing vulnerability and limitations within these systems. Over a year later, we can conclude that neither governments, healthcare systems, nor healthcare professionals were prepared for such a scenario. Globally, patients with pre-existing psychiatric disorders experience an exacerbation of psychiatric symptoms triggered by social distancing, isolation, and the loss or fear of losing their jobs [1]. They become more anxious, angry, stressed, agitated, and withdrawn during the pandemic outbreak or in quarantine [2]. The general public was also not affected by the pandemic, with lower psychological well-being and higher scores of anxiety and depression in the general public [1]. A systematic review of data both from scientific and non-scientific sources in several languages from 28 countries found numerous reports of deteriorations in symptoms, impacts of loneliness and social isolation, and lack of access to services and resources [3]. On the other hand, they also observed examples of resilience, effective self-management, and peer support (ibid). In a study performed among 23 European experts in the early phase of the pandemic, it was found that some measures implemented by state authorities led to elevated stress levels, depression, domestic violence, and suicide among the inhabitants of involved countries [4].

Changes in the functioning of mental health services were also observed. Healthcare providers postponed several routines, elective care, and outpatient services [5, 6]. 
Face-to-face consultations have been disrupted because of hesitation in consulting doctors in a hospital setting. As per a WHO survey conducted in 155 countries, the majority of countries have reported partial or complete disruption of healthcare services for non-COVID diseases [7]. Due to the pandemic, only the most affected patients, i.e., suicidal, psychotic, or delirious, access public health facilities through emergency wards as the activity of many outpatient clinics was discontinued [4]. The pandemic led to a drastic reduction in levels of care in community mental health centers and general hospital psychiatric wards [8], including treatment refusal by the patients, difficulty complying with safety precautions due to psychosis, agitated behavior, and problems with staff psychological well-being [9]. Schizophrenia is a prototypical severe mental illness in which the insufficiency of the existing medical care system can be traced. It is known that patients with schizophrenia are at a higher risk of developing respiratory diseases, including more severe forms of COVID-19 [10]. Accordingly, people with this diagnosis during a pandemic should have received more care and attention. Unfortunately, it did not happenpatients had difficulty getting in touch with a doctor let alone accessing better care and prevention [11].

The COVID-19 pandemic has put healthcare professionals across the world in an unprecedented situation, having to make difficult decisions, e.g., how to allocate scant resources to equally needy patients, how to balance their own physical and mental healthcare needs with those of patients, how to align their desire and duty to patients with those to family and friends, and how to provide care for all severely unwell patients with constrained or inadequate resources [12]. Accordingly, healthcare professionals reported increased depressive symptoms, anxiety, mental illness, and poor sleep quality [1]. The studies on the impact of the COVID-19 pandemic on mental health are ongoing [13]. A profound understanding of the impact of the pandemic could facilitate an optimized governmental, social, and individual health preparedness during future pandemics and/or natural disasters. It may lead to coordinated actions through multi-level guideline development with the aim to improve mental health outcomes globally.

During the course of the pandemic, telecommunications technologies, telemedicine, have played a critical role in the provision of medical care in general and psychiatric care specifically as both widespread and sporadic quarantines and lockdowns in response to COVID-19 limited access to inperson care. It has proved to be beneficial, cost-efficient, and satisfactory for patients and providers across various disease types, unless the need for physical examination becomes imperative [14]. For example, the New York-Presbyterian/ Weill Cornell Medical Center reported an increase of $8729 \%$ in the use of video visits during the COVID-19 period as compared with the pre-COVID-19 period [15], and increase seen across both individual providers, organizations, and systems. In psychiatry, an American Psychiatric Association (APA) survey conducted at the beginning of the pandemic reported that most psychiatrists (64\%) were using no telehealth prior to the pandemic with a shift to $85 \%$ seeing more than $75 \%$ of their patients via telehealth in 2 months. Numerous reports have been published describing lessons learned from psychiatric organizations' rapid virtualization and conversion to telehealth operations emphasizing how telehealth was a critical lifeline in maintaining services and access to care during the pandemic [16-18]. It is important to understand the trends in telecommunications technologies leading up to and impacting their widespread use in the pandemic, current lessons learned, and implications for global mental health systems for both emerging from the pandemic and after the pandemic has ended. This information with particular attention to impacts on policy issues is critical in shaping the future direction of Global Mental Health Care. Below, we summarize the trends in mental health telecommunications (e-mental health) leading up to and in the initial phase of the pandemic and current trends synthesizing the policy implications and recommendations for mental health providers and organizations.

\section{e-Mental Health}

e-Mental health (eMH) refers to the use of telecommunication technologies for the treatment or prevention of mental health disorders (Mucic D, Hilty DM, 2016). Telepsychiatry (i.e., real-time video conferencing) is the longest known and best evidence-based eMH application. It has certainly also shown its potential during the current pandemic $[19,20]$.

Mobile health (mHealth) and other technology-based services facilitate mental health service delivery and may be considered part of an eMH spectrum of care [21]. Virtual reality applications are used in the treatment of PTSD, specific phobias, anorexia, autism spectrum disorders, and schizophrenia [22, 23]. In 2019, researchers identified 1435 mobile apps for treating anxiety disorders, depression, schizophrenia, self-harm, and substance abuse, although the number is increasing every year [23]. Smartphone-based mobile apps have contributed significantly to the development of personalized psychiatry.

New technologies associated with artificial intelligence (AI) are machine learning (ML) and deep learning in a form of convolutional neural networks (CNNs). ML and CNNs allow for an earlier and more precise diagnosis of the patient, the determination of the risk of deterioration or suicidal behavior, and the prediction of the therapeutic effect based on the analysis of the earlier effectiveness of drugs in patients with similar symptom clusters and the prediction of long-term effects such as relapse, side effects, or health complications [24-26]. In the future, AI may become a technology that supports physicians in diagnosis, drug selection, 
and prediction of improvement outcome. The possibilities that AI offers, based on the analysis of the collected records, are unpredictable and limitless.

In the case of mentally ill individuals, however, the current pandemic was a turning point for primarily telepsychiatry, while various eMH applications followed in addition to either personal contact with the professionals, i.e., hybrid care models (i.e., in-person and/or technology combinations), or "remote" consultation or as a part of self-directed assessment and care. On a spectrum of low to high engagement and technology requirements, participants use website information, support and chat groups, social media, resources for self-directed assessment and care, asynchronous patient-clinician communication (i.e., mobile health app, text, or e-mail), video, and hybrid care models [27, 28]. While it is enjoyable and helpful to be spontaneous in using technology, it is helpful to consider the source used (e.g. Internet, social media, e-consultation, video), the user's goal/aim, liabilities, and approaches to make this meaningful and prudent (Table 1). For example, easy-to-use options like chat rooms or social media may vary in quality, yet largely offer a positive form of support for a patient, spouse, or caregiver, yet more meaningful change may require an in-depth evaluation and clinical relationship via video.

\section{Shifts in Moving to Care With Asynchronous Technologies}

Clinical care in-person, by telepsychiatry, and via asynchronous technologies have significant things in common, but there are differences, too $[29,30]$. There are paradigm shifts happening with technology-based care. One shift is moving from structural (e.g., office visit) to functional "in time" clinical care. New research is focusing on how technology affects the therapeutic frame, communication, boundaries, and trust by applying neuroscience, communication, business, and augmented and virtual reality literature to psychiatry [31]. Correspondence via technology outside the office visit creates a new, previously impossible, sense of continuity, connection, and ease of communication (ibid.). Another shift is "intime" data collection with wearables and sensors-for heart rate, steps/activity, and other parameters in older adults-is known as ecological momentary assessment (EMA) yields more valid, reliable, and meaningful data [32] - this empowers clinician decision-making (e.g., mood and medication management). To use that data, though, mobile health, video, social media, and other technologies have to be integrated into the clinical workflow - this requires administrative planning, training, and faculty development [33].

\section{Competencies for Video and Other Technologies}

As new generations adapt smartphones, social media, and other technologies, they have more personal technology experience than their teachers [33]. Many professionals may not consider asynchronous or store-and-forward technologies like mobile health and social media as a part of care, but patients do [29]. To keep up with society's use of technology and to ensure quality care, clinicians need skills (i.e., competencies), knowledge, and attitudes for synchronous and asynchronous technologies [30, 34]. Competencybased education focuses on clinical skill development in addition to knowledge acquisition. Competencies dovetail with lifelong learning as a part of ongoing practice, but clinicians are pushed toward quality care for many other reasons. Clinical experience-propelled forward by COVID19-appears to predict interest and level of concerns. Perhaps, conservatively a dose of $1-5 \mathrm{~h}$ is needed to debunk preliminary questions/concerns or $6-10+\mathrm{h}$ to gain skills [35]. Competencies for video and other technologies have been organized into the six Accreditation Council of Graduate Medical Education (ACGME) domains [36], which are Patient Care, Medical Knowledge, System-Based Practice, Professionalism, Practice-Based Learning and Improvement, and Interpersonal and Communication Skills. Using the ACGME domains but in the specific area of mobile health, the Patient Care competency mainly includes history taking, engagement and interpersonal skills, assessment, and management. It also includes administration, documentation, and medico-legal issues such as privacy, confidentiality, safety, data protection/integrity, and security. In general, clinicians should help patients and trainees reflect on the pros/cons of mobile phones, apps, and other eMH applications. A competent/proficient clinician selects the eMH option based on patient preference, skill, and need (i.e., purpose) so that patient and clinician decisions in-time are enhanced. It is helpful to know if the patient uses e.g. videoconferencing, apps, or texts for personal life, health care, and/or behavioral health care, and if the patient is aware of risks (e.g., privacy, security).

\section{Virtual Hybrid Provider-Patient Relationships in e-Mental Health}

Over the past decade, the concept of hybrid psychiatrist/ provider-patient relationships has emerged [37]. With the increasing use of technology in medicine and psychiatry, the provider-patient relationship is now managed across multiple settings and platforms including in-person, videoconferencing, patient portals, mobile devices, websites, and social networks. Providers need to be able to effectively work with an individual patient across these settings with and appreciation of the strengths, challenges, nuances, and need for adapting clinical styles and processes to best fit an individual patient's circumstances, problems, and environment [38]. The trend to work with patients in hybrid manners was greatly accelerated by the 


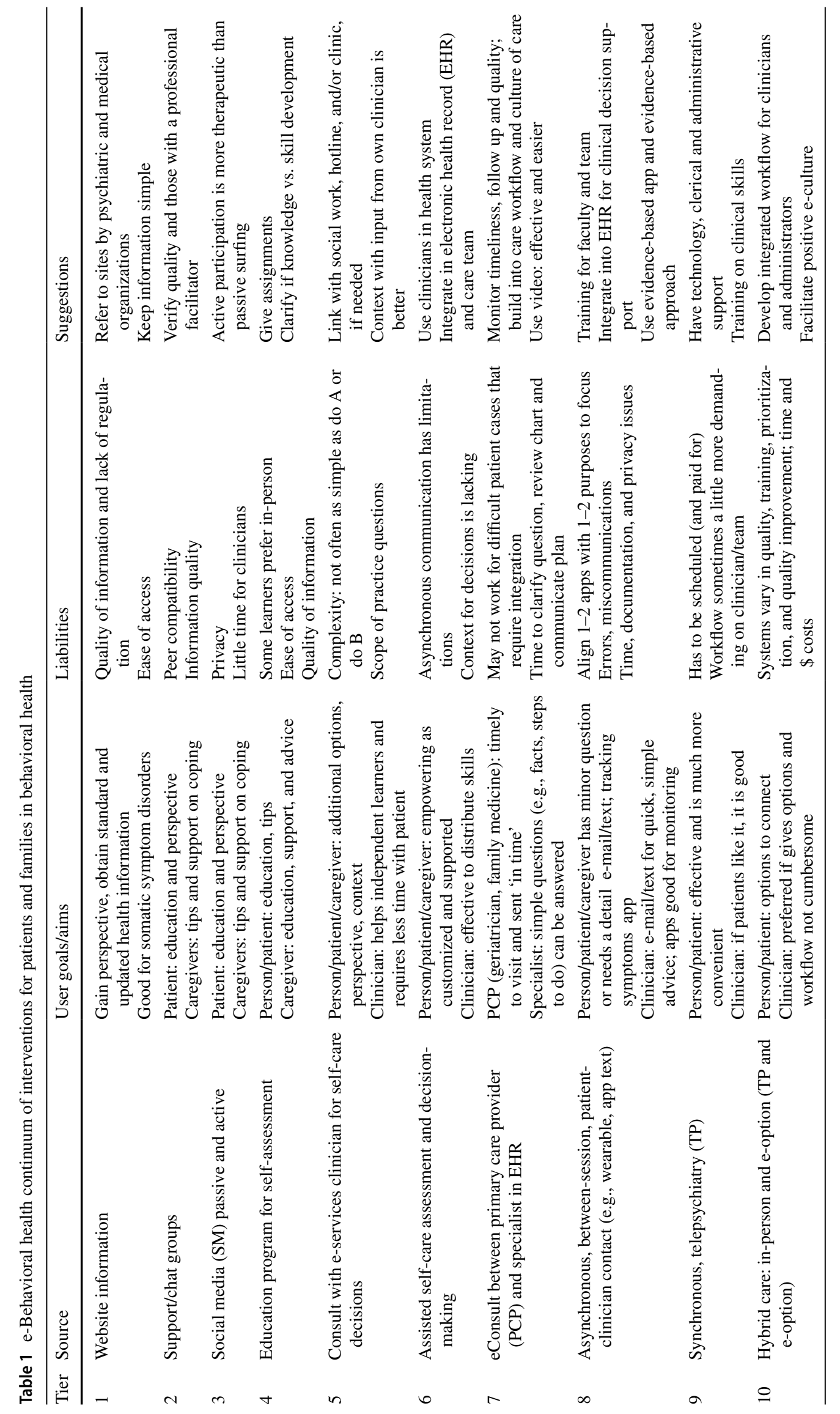


COVID pandemic, necessitating individual providers to increase their mastery of hybrid relationships as well as provide an impetus for the field to gain a deeper understanding of the utility and impact on provider-patient relationships of commonly used technology.

Technology may change the nature of interaction for participants and communication related to the exchange of information, clarity, responsiveness, and comfort [33]. The goal with technology is to simulate real-time experiences related to feelings, perception, images, and interaction. Telepsychiatry, practiced via standardized, remote control, i.e., pan-tilt zoom video equipment, is closest to the "golden standard" for personal consultations. Even low-cost, videoplatform-based telepsychiatry facilitates engagement and a "social presence." Ultimately, the goal via any modality is to create an environment that facilitates the therapeutic engagement and emotional well-being for all parties [34].

\section{Telemedicine Psychiatric Systems}

Global consensus and national policies have emphasized deinstitutionalization, or a shift in providing mental health care from institutional to community settings. The integration of psychiatry with somatic medicine and the development of a community psychiatry requires the organization and creation of a complex and coordinated care system, including primary care, specialized mental health care, crisis management services, housing programs, inpatient units, and centers running a specialized treatment program and supervising this system and consulting them clinical academic centers [11]. This creates great opportunities to introduce innovative technologies in the form of telemedicine systems coordinating the operation of a multi-module care system. Such a telemedicine system may include other mobile medical applications that enable screening diagnosis, early diagnosis, cognitive training, video education, and digital forms of treatment with the use of virtual reality, augmented reality, and applications for relaxation or phonotherapy $[39,40]$. Such systems can automatically accelerate patient care by monitoring symptoms and processing them through algorithms that prioritize patients with more severe mental health problems [11, 41].

\section{(e-)Mental Health Care and Future Directions of Global Mental Health Care}

We must recognize that the global COVID-19 pandemic will most likely be followed by a secondary pandemic of mental and behavioral illness and we need to implement steps to mitigate the impact of COVID on global mental health [42]. With the widespread workforce shortage of psychiatrists and mental health providers prior to COVID-19, it is imperative to discuss how the mental healthcare systems will manage future unprecedented events. One way that mental healthcare providers can accommodate an increasing demand for mental health services is through the increased use of eMH within and across national borders. Increasing the use and availability of eMH applications and online resources is one way in which we can prepare for the growing demand for psychiatric services during and after the pandemic. As $45 \%$ of the world's population has access to 1 psychiatrist for every 100,000 people, and $50 \%$ of the population has a smartphone, the potential of providing psychiatric care through mobile applications is significant, especially with an emphasis on population health-based approaches [43]. Increased adoption, competencies, and investments in digital health today lay the groundwork of increased access and use of mental health care of the future [44].

However, in this exciting era of innovation and the adoption of new technologies in mental health care, many tensions have arisen including those of innovation vs regulation, access vs evidence, monetization vs privacy, and pragmatism vs rigor. COVID-19 has resulted in a loosening of regulations impacting e-mental health, which has allowed easier access to care. These rules often well-intentioned can create barriers and limit access to care. Overall, the benefits of access outweigh many of the concerns of technology in mental health care that the regulations have attempted to address. Patients, providers, and many health systems for sure do not want to go back to pre-COVID circumstances but it is unclear what the right balance between in-person and e-mental health treatment is and what the optimal balance of hybrid relationships is. Ultimately, the solution is one that prioritizes the individual patient's needs taking into account the context of their environment and treatment systems. The following actors play a crucial for the future of (e)-mental health care:

1. Patients are the ones who have the most stake in the tools used for their treatments and have individual mediated levels of comfort and access with technologies that impact their ability to engage in e-mental health

2. Clinicians are the ones who use the technology and require a good understanding of available technology's capabilities and the relevant competencies to use these in treatment.

3. Health systems provide the structure and environments for clinical engagement and drive the decision on which tools are used and require appropriate information (e.g., evidence, ROI) to make systemwide decisions.

4. Decision-makers need to coordinate at the national and global levels to facilitate implementation, collaboration, and engagement in eMH (e.g., exchange of expertise and resources across national borders). 


\section{Globalization of eMH}

In order to prepare for future unprecedented events (i.e., pandemics and/or natural disasters), one of the main goals now should be to create national as well as international, multilingual medical platforms and processes that facilitate access to medical services [45]. This requires consideration of telemedicine as an integral part of the healthcare system and the creation of telemedicine systems that integrate different medical specialties and provide interoperability to their users - both healthcare professionals and patients.

eMH is unfortunately still an underrepresented topic globally and plays a minor to moderate role in (national) mental health policies. Globally, the main limiting factors on the sustainable implementation of telemedicine are infrastructure, costs, lack of digital literacy, and staff resistance to change $[45,46]$. There are current significant barriers to global collaboration which include the global regulation of the use of various eMH applications not only within national borders but largely across national borders. The analysis of the current development of eMH in the world indicates the need to create an international framework and uniform standards for telemedicine that are currently lacking. "Transnational Policy for e-Mental Health," a guidance document published last year, aimed to foster the potential for adoption and implementation of eMH solutions throughout Europe [47].

There has been a steady increase in global migration and immigration as people are now moving further, faster, and in greater numbers than ever before propelled on by a number of interacting and complex factors including conflict, economics, and education [48]. Many of migrants and immigrants who have a potential need for mental health assistance can benefit from technology that knows no borders [49]. Unfortunately, current local licensure regulations are still an obstacle to international collaboration and expansion of the scope of digital services. Concerns related to e.g. telepsychiatry are primarily based on rules regarding the patient's location and the professional's, respectively. It is common that the patient cannot be treated if not located within the same country boundaries as the professional. The World Psychiatric Organization (WPA) recently took specific steps in support of "telepsychiatry globalization" publishing the first global guidelines, aimed at promoting and facilitating telepsychiatry services capable of providing remote consultations across national borders [50]. The document refers, among other things, to an interesting development in Greece where the majority of telepsychiatry consultations are stored using cloud technology. In Greece, the consultation adheres to the rules and regulations valid in the country where the"cloud server" is located. Accordingly, the telepsychiatry provider must be certified in the country where the cloud server is located, i.e., not where the patient is located. Although this can present some challenges around service location, the conceptualization of the visit with the doctor "up in the cloud" addresses some of the challenges of needing to be licensed where a patient is physically located at the time of a clinical session. This change could serve as a template for others to change/modify existing jurisdictional regulations.

The increased need for just-in-time information about both the technical aspects, implemenation, and effectiveness of e-mental health is vital in helping the key actors make appropriate decisions and planning for e-mental health. Our current models of research and development and information dissemination were not keeping paced and were not designed for an era of rapid technological change that has which has accelerated even more during the COVID pandemic. Only about half of evidence-based practice ever achieve widespread dissemination in health care taking an average of 17 years to be incorporated into routine practice [51]. There is an urgent need to develop new R\&D models that can more rapidly evaluate $\mathrm{eMH}$ applications and services including critical lessons currently being learned during the COVID pandemic.

Addressing these regulatory, policy, and evaluation challenges could have significant benefits for global e-mental health for:

1. Patients. The ability to reach out to providers in other countries who have more availability and cultural understanding of their backgrounds reduces waiting times and travel. For those patients who work, study, or travel abroad, the opportunity to maintain continuity in care despite distance will be greatly appreciated. The current EU refugee crisis is perfectly positioned to benefit from an international network of bilingual experts aimed at assessing and/or treating refugees and migrants via telepsychiatry without the use of interpreters, i.e., via mother tongue.

2. Clinicians. Availability of timely qualified "second opinion" from colleagues with a relevant cultural and linguistic background regarding assessment and/or treatment of ethnic minorities with limited language proficiency. Furthermore, international expertise may be brought via telepsychiatry to local health workers as a part of education, supervision, and scientific collaboration.

3. Health systems. Increase their quality of care and address greater potential health through improving access to care in mental health systems.

4. For decision-makers. Raise the quality of national health care, be better prepared to respond to future "unprecedented events," and widen participation in the global community. 


\section{Conclusions}

The experiences of the COVID-19 pandemic will undoubtedly affect and shape the future of (mental) health care globally. In the face of the pandemic, digital technology-based approaches are increasingly being emphasized. This global crisis offers the opportunity to advance our understanding of how to develop models of "traditional services with modern approaches" through digital technologies. Wider understanding and the use of the new technologies allow many mentally ill individuals to receive high-quality treatment and care that otherwise remain inaccessible. eMH improves access, reduces costs, and compliments other care options. Regulatory constraints, however, currently pose significant barriers to the broader adoption of eMH within and across country borders. The collaborative development of global standards along with policies and appropriate regulations opens the possibility of improved access to care, including the free choice of healthcare providers, even across national boundaries. For these reasons, it is important to make a start in developing a global perspective for this work in the field of eMH. It is partially dependent on policymakers' insight and willingness to act promptly. Efforts such as the WPA's "Telepsychiatry Global Guidelines" along with "Transnational Policy for e-Mental Health" can pave the way for improved international cooperation. These can provide an example for decision-makers to inspire steps towards unifying rules and global regulations for the entire eMH spectrum in order to break down the barriers of accessibility. Reaching a common understanding of the appropriate standards, policies, and medico-legal requirements for the use of eMH, while considering its constant technological and methodological upgrading, is likely to make a crucial contribution to reducing global disparity in access to care. Additional obstacles related to lack of expertise and/or a lack of clinical experience can be addressed by making sure clinicians and systems have the tools and information with appropriate training and guidance related to the selection of video tools as well as their proper use. Limitation drives innovation. Identifying the problem is the first step towards the solution. The Global Community must act more aggressively to improve access to qualified care for those who are most vulnerable in these very challenging times.

Acknowledgements The editors would like to thank Dr. Robert Caudill for taking the time to review this manuscript.

\section{Compliance with Ethical Standards}

Conflict of Interest Mucic D., Hilty DM, Krzystanek M, and Krysta $\mathrm{K}$ each declare no potential conflicts of interest. Dr. Shore is Chief Medical Officer at AccessCare, a provider of telehealth services and technologies.
Human and Animal Rights and Informed Consent This article does not contain any studies with human or animal subjects performed by any of the authors.

\section{References}

Papers of particular interest, published recently, have been highlighted as:

- Of importance

1. Vindegaard N, Benros ME. COVID-19 pandemic and mental health consequences: systematic review of the current evidence. Brain Behav Immun. 2020;89:531-42. https://doi.org/10.1016/j. bbi.2020.05.048.

2. Torales J, O'Higgins M, Castaldelli-Maia JM, et al. The outbreak of COVID-19 coronavirus and its impact on global mental health. Int J Soc Psychiatry. 2020;66(4):317-20. https://doi.org/10.1177/ 0020764020915212.

3. Sheridan Rains L, Johnson S, Barnett P, et al. Early impacts of the COVID-19 pandemic on mental health care and on people with mental health conditions: framework synthesis of international experiences and responses. Soc Psychiatry Psychiatr Epidemiol. 2021;56(1):13-24. https://doi.org/10.1007/s00127-020-01924-7.

4. Thome J, Deloyer J, Coogan AN, et al. The impact of the early phase of the COVID-19 pandemic on mental-health services in Europe. World J Biol Psychiatry. 2020;23:1-10. https://doi.org/ 10.1080/15622975.2020.1844290.

5. World Health Organization. COVID-19: operational guidance for maintaining essential health services during an outbreak: interim guidance, 25 March 2020. Available at https://apps.who.int/iris/ handle/10665/331561.

6. World Health Organization. Weekly operational update on COVID19, 4 September 2020. Available at https://www.who.int/docs/ default-source/coronaviruse/situation-reports/wou-4-september2020-approved.pdf?sfvrsn=91215c78_2.

7. World Health Organization (WHO). COVID-19 significantly impacts health services for noncommunicable diseases. June, 2020 (Available from: )https://www.who.int/news/item/0106-2020-covid-19-significantly-impacts-health-services-fornoncommunicable-diseases.

8. Carpiniello B, Tusconi M, Zanalda E, et al. Psychiatry during the Covid-19 pandemic: a survey on mental health departments in Italy. BMC Psychiatry. 2020;20(1):593. https://doi.org/10.1186/ s12888-020-02997-z.

9. Mahgoub N, Agarkar S, Radosta M, et al. Inpatient psychiatry unit devoted to COVID-19 patients. Compr Psychiatry. 2021;9(107): 152237. https://doi.org/10.1016/j.comppsych.2021.152237.

10. Fonseca L, Diniz E, Mendonça G, Malinowski F, Mari J, Gadelha A. Schizophrenia and COVID-19: risks and recommendations. Braz J Psychiatry. 2020;42(3):236-8. https://doi.org/10.1590/ 1516-4446-2020-0010.

11. Mari JJ, Gadelha A, Kieling C, Ferri CP, Kapczinski F, Nardi AE, Almeida-Filho N, Sanchez ZM, Salum GA. Translating science into policy: mental health challenges during the COVID-19 pandemic. Braz J Psychiatry. 2021;12:S1516-44462021005004200. https://doi.org/10.1590/1516-4446-2020-1577.

12. Esterwood E, Saeed SA. Past epidemics, natural disasters, COVID19, and mental health: learning from history as we deal with the present and prepare for the future. Psychiatr Q. 2020;91:1121-33. https:// doi.org/10.1007/s11126-020-09808-4.

13. Agorastos A, Tsamakis K, Solmi M, Correll CU, Bozikas VP. The need for holistic, longitudinal and comparable, real-time 
assessment of the emotional, behavioral and societal impact of the COVID-19 pandemic across nations. Psychiatrike $=$ Psychiatriki. 2021 Mar. https://doi.org/10.22365/jpsych.2021.010.

14. Nanda M, Sharma R. A review of patient satisfaction and experience with telemedicine: a virtual solution during and beyond COVID-19 pandemic. Telemed J E Health. 2021. https://doi.org/ 10.1089/tmj.2020.0570.

15. Ramaswamy A, Yu M, Drangsholt S, et al. Patient satisfaction with telemedicine during the COVID-19 pandemic: retrospective cohort study. J Med Internet Res. 2020;22:e20786.

16. Connolly SL, Stolzmann KL, Heyworth L, Weaver KR, Bauer MS, Miller CJ. Rapid increase in telemental health within the Department of Veterans Affairs during the COVID-19 pandemic. Telemedicine and e-Health. 2021;27(4):454-8.

17. Mishkind MC, Shore JH, Bishop K, D'Amato K, Brame A, Thomas M, Schneck CD. Rapid conversion to telemental health services in response to COVID-19: experiences of two outpatient mental health clinics. Telemed J E Health. 2020.

18. Yellowlees P, Nakagawa K, Pakyurek M, Hanson A, Elder J, Kales HC. Rapid conversion of an outpatient psychiatric clinic to a $100 \%$ virtual telepsychiatry clinic in response to COVID -19. Psychiatr Serv. 2020;71(7):749-52.

19. O'Brien M, McNicholas F. The use of telepsychiatry duringCOVID-19 and beyond. Ir J Psychol Med. 2020;37(4):250-5. https://doi.org/10.1017/ipm.2020.54.

20. Haxhihamza K, Arsova S, Bajraktarov S, et al. Patient satisfaction with use of telemedicine in university clinic of psychiatry: Skopje, North Macedonia during COVID-19 pandemic. Telemed J E Health 2020. https://doi.org/10.1089/tmj.2020.0256.

21. Hilty DM, Chan S, Hwang T, Wong A, Bauer AM. Advances in mobile mental health: opportunities and implications for the spectrum of e-mental health services. Focus (Am Psychiatr Publ). 2018;16(3):314-27.

22. Park MJ, Kim DJ, Lee U, Na EJ, Jeon HJ. A literature overview of virtual reality (VR) in treatment of psychiatric disorders: recent advances and limitations. Front Psychiatry. 2019;19(10):505. https://doi.org/10.3389/fpsyt.2019.00505.

23. Larsen ME, Huckvale K, Nicholas J, Torous J, Birrell L, Li E, Reda B. Using science to sell apps: evaluation of mental health app store quality claims. NPJ Digit Med. 2019;22(2):18. https:// doi.org/10.1038/s41746-019-0093-1.

24. Ibrahim FA, Pahuja E, Dinakaran D, Manjunatha N, Kumar CN, Math SB. The future of telepsychiatry in India. Indian J Psychol Med. 2020;42(5 Suppl):112S-117S. https://doi.org/10.1177/ 0253717620959255.

25. Bzdok D, Meyer-Lindenberg A. Machine learning for precision psychiatry: opportunities and challenges. Biol Psychiatry Cogn Neurosci Neuroimaging. 2018;3(3):223-30. https://doi.org/10. 1016/j.bpsc.2017.11.007.

26. Rajkomar A, Oren E, Chen K, et al. Scalable and accurate deep learning with electronic health records. NPJ Digit Med. 2018;8(1):18. https://doi.org/10.1038/s41746-018-0029-1.

27. Hilty DM, Chan S, Torous J, et al. New frontiers in healthcare and technology: Internet- and web-based mental options emerge to complement in-person and telepsychiatric care options. J Health Med Inform. 2015;6(4):1-14.

28. Chan S, Godwin H, Gonzalez A, et al. Review of use and integration of mobile apps into psychiatric treatments. Curr Psychiatry Rep. 2017;19(12):96. https://doi.org/10.1007/s11920-017-0848-9.

29. Zalpuri I, Liu H, Stubbe D, et al. A competency-based framework for social media for trainees, faculty and others. Acad Psychiatry. 2018;42(6):808-17.

30. Hilty DM, Uno J, Chan S, et al. Role of technology in professional development. Psych Clin N Amer. 2019;42:493-512.
31. Hilty DM, Randhawa K, Maheu MM, et al. A review of telepresence, virtual reality and augmented reality applied to clinical care. J Tech Behav Sci. 2020. https://doi.org/10.1007/s41347-020-00126-x.

32. Hilty DM, Armstrong CM, Luxton DD, et al. A framework of sensor, wearable and remote patient monitoring competencies for clinical care and training: scoping review. J Tech Behav Sci. 2021. https://doi.org/10.1007/s41347-020-00190-3.

33. Hilty DM, Torous J, Parish M, et al. A literature review comparing clinicians' approaches and skills to in-person, synchronous and asynchronous care: moving toward asynchronous competencies to ensure quality care. Telemed J E-Health. 2020. https://doi.org/ 10.1089/tmj.2020.0054.

34. Maheu M, Drude K. Hertlein K, et al. An interdisciplinary framework for telebehavioral health competencies. J Tech Behav Sci. 2019;3(2):108-40; correction 3(2): 107.

35. Cruz C, Orchard K, Shoemaker EZ, Hilty DM. A survey of residents/fellows, program directors and faculty about telepsychiatry: clinical experience, interest, views and misconceptions. J Tech Behav Sci. 2021. https://doi.org/10.1007/s41347-021-00201-x.

36. Accreditation Council on Graduate Medical Education. Common Program Requirements, 2013. https://www.acgme.org/acgmeweb/ Portals/0/PFAssets/ProgramRequirements/CPRs2013.pdf.

37. Yellowlees P, Shore JH. Telepsychiatry and health technologies: a guide for mental health professionals. American Psychiatric Pub; 2018 Jan 22.

38. Shore JH. Managing virtual hybrid psychiatrist-patient relationships in a digital world. JAMA Psychiat. 2020;77(5):541-2.

39. Krzystanek M, Krysta K, Borkowski M, et al. The effect of smartphone-based cognitive training on the functional/cognitive markers of schizophrenia: a one-year randomized study. J Clin Med. 2020;9(11):3681. https://doi.org/10.3390/jcm9113681.

40. Schuster I, Romańczyk M, Surma S, Krzystanek M. Phonotherapy - a new biological treatment proposal for the augmentation of pharmacotherapy in psychiatry and neurology. Adv Psychiatry Neurol. 2020;29(4):224-33. https://doi.org/10.5114/ppn.2020. 103634.

41. Miyoshi NSB, Azevedo-Marques JM, Alves D, Azevedo-Marques PM. An eHealth platform for the support of a Brazilian Regional Network of Mental Health Care (eHealth-Interop): development of an interoperability platform for Mental Care Integration. JMIR Ment Health. 2018;5(4): e10129. https://doi.org/10.2196/10129.

42. Galea S, Merchant RM, Lurie N. The mental health consequences of COVID-19 and physical distancing: the need for prevention and early intervention. JAMA Intern Med. 2020;180(6):817-8. https:// doi.org/10.1001/jamainternmed.2020.1562.

43. Lovejoy CA, Buch V, Maruthappu M. Technology and mental health: the role of artificial intelligence. Eur Psychiatry. 2019;55:1-3. https://doi.org/10.1016/j.eurpsy.2018.08.004.

44. Torous J, Jän Myrick K, Rauseo-Ricupero N, Firth J. Digital mental health and COVID-19: using technology today to accelerate the curve on access and quality tomorrow. JMIR Ment Health. 2020;7(3): e18848. https://doi.org/10.2196/18848.

45. Scott Kruse C, Karem P, Shifflett K, Vegi L, Ravi K, Brooks M. Evaluating barriers to adopting telemedicine worldwide: a systematic review. J Telemed Telecare. 2018;24(1):4-12. https://doi.org/ 10.1177/1357633X16674087.

46. Sagaro GG, Battineni G, Amenta F. Barriers to sustainable telemedicine implementation in Ethiopia: a systematic review. Telemed Rep. 2020;1(1):8-15. https://doi.org/10.1089/tmr.2020.0002.

47. Gaebel W. et al. Transnational Policy for e-Mental Health. March 2020. Available at https://www.nweurope.eu/media/ 10450/emen_transnational-policy-for-e-mental-health guidance-document_3-2020.pdf. The Transnational Policy for e-Mental Health is a document aiming to increase the implementation of high-quality e-mental health (eMH) and 
pave the way for broader international collaboration within the field of eMH in EU.

48. Rousseau C. Addressing mental health needs of refugees. The Canadian Journal of Psychiatry. 2018;63(5):287-9.

49. Reinhardt I, Gouzoulis-Mayfrank E, Zielasek J. Use of telepsychiatry in emergency and crisis intervention: current evidence. Curr Psychiatry Rep. 2019;21(8):1-8.

50. WPA Telepsychiatry Global Guidelines, 2021. Available at https:// 3ba346de-fde6-473f-b1da-536498661f9c.filesusr.com/ugd/ e172f3_19ce700c2a1f484b98efdcaf02c3c6ff.pdf. WPA Global Telepsychiatry Guidelines aims to pave the way to increased international collaboration of policymakers too and inspire them to make a step forward toward unification of regulations enabling easier collaboration and access to care regardless of the national borders.

51. Bauer MS, Damschroder L, Hagedorn H, Smith J, Kilbourne AM. An introduction to implementation science for the non-specialist. BMC psychology. 2015;3(1):1-2.

Publisher's Note Springer Nature remains neutral with regard to jurisdictional claims in published maps and institutional affiliations. 